\title{
Sleep-Disordered Breathing in Patients With Idiopathic Cardiomyopathy
}

\author{
Katsuhisa Banno, MD; Toshiaki Shiomi, MD; Ryujiro Sasanabe, MD; Kazuo Otake, MD; \\ Rika Hasegawa, MD; Masato Maekawa, MD*; Takayuki Ito, MD
}

\begin{abstract}
Background Sleep-disordered breathing may adversely affect heart function, and thereby contribute to the progression of heart failure. A study was undertaken in patients with idiopathic cardiomyopathy to document the characteristics of sleep-disordered breathing.

Methods and Results Thirty-five patients with a diagnosis of idiopathic cardiomyopathy, comprising 20 patients with dilated cardiomyopathy (DCM) and 15 patients with hypertrophic cardiomyopathy (HCM), underwent overnight polysomnography. Of these 35, 16 (80\%) of the DCM patients and 7 (47\%) of the HCM patients had sleep-disordered breathing. Central sleep apnea-hypopnea syndrome (CSAHS) was seen in 10 DCM patients, but not in the HCM patients, and obstructive sleep apnea-hypopnea syndrome (OSAHS) was seen in 6 DCM patients and 7 HCM patients. CSAHS was seen in DCM patients with a low left ventricular ejection fraction. HCM patients with OSAHS had a significantly greater body mass index (BMI) than those without OSAHS and CSAHS $\left(27.6 \pm 3.8\right.$ vs $\left.22.0 \pm 4.0 \mathrm{~kg} / \mathrm{m}^{2}, \mathrm{p}<0.05\right)$. DCM patients with OSAHS had a larger BMI than those with CSAHS $\left(29.3 \pm 5.8\right.$ vs $\left.24.2 \pm 4.0 \mathrm{~kg} / \mathrm{m}^{2}, \mathrm{p}<0.05\right)$ and those without OSAHS and CSAHS $(29.3 \pm 5.8$ vs $\left.21.3 \pm 3.1 \mathrm{~kg} / \mathrm{m}^{2}, \mathrm{p}<0.05\right)$.

Conclusions Sleep-disordered breathing is common in patients with idiopathic cardiomyopathy; half of DCM patients had CSAHS, which was closely associated with obesity. (Circ J 2004; 68: 338-342)
\end{abstract}

Key Words: Apnea; Cardiomyopathy; Obesity; Sleep

C ardiomyopathy is defined as a primary disorder of the myocardium associated with cardiac dysfunction. There are various classifications of cardiomyopathy, among which the new WHO classification is widely recognized. The functional classification includes 5 categories: (1) dilated cardiomyopathy (DCM), (2) hypertrophic cardiomyopathy (HCM), (3) restrictive cardiomyopathy, (4) arrythmogenic right ventricular cardiomyopathy and (5) unclassified cardiomyopathies! DCM and HCM are relatively common and linked to the complications of sudden death, heart failure, arrhythmia. Sudden death can occur during the night in patients with DCM or HCM, whereas heart failure can become worse because of nocturnal hypoxemia. It is possible that sleep-disordered breathing has some deleterious effect on patients with DCM or HCM during the night. Recent studies show a relationship between sleep-disordered breathing and DCM;2-12 however, little is known about the characteristics of sleep-disordered breathing in HCM patients,$^{13}$ or the differences in sleep-disordered breathing between DCM and HCM. To investigate the characteristics of sleep-disordered breathing in Japanese patients with idiopathic cardiomyopathy, we studied 35 patients without hypertension.

(Received January 16, 2003; revised manuscript received January 22, 2004; accepted January 27, 2004)

Sleep Disorders Center and Department of Cardiology and *Department of Internal Medicine, Aichi Medical University School of Medicine, Aichi, Japan

Mailing address: Katsuhisa Banno, MD, Sleep Disorders Center, Aichi Medical University Hospital, 21 Karimata, Yazako, Nagakutecho, Aichi-gun, Aichi 480-1195, Japan. E-mail: katsu-aic@umin.ac. jp

\section{Methods}

Patients

The subjects were 35 patients diagnosed as having idiopathic cardiomyopathy (20 DCM; $15 \mathrm{HCM}$ ) in the cardiology division of Aichi Medical University Hospital from 1990 to 2001. There were 17 men and 3 women with DCM, and 10 men and 5 women with HCM. The definition and classification of cardiomyopathy was based on the report of the 1995 World Health Organization/International Society and Federation of Cardiology Task Force!

All patients were evaluated by cardiologists to confirm that they were clinically stable and on standard therapy, with stable heart failure within 4 weeks before enrollment. Twenty-five patients had been on diuretics, 15 on angiotensin-converting enzyme inhibitors and 2 on angiotensin II receptor antagonists, and isosorbide dinitrate was used in 10 , Ca blockers in $8, \beta$-blockers in 16 and digoxin in 4 . We excluded patients who had hypertension, acute congestive heart failure, acute pulmonary edema, intrinsic pulmonary disease, intrinsic renal and liver disease, and intracranial disease.

\section{Sleep Studies}

The 35 patients underwent overnight polysomnography by the conventional method. Continuous recordings were made with a computerized system (Sleep Watcher ${ }^{\mathrm{TM}}$; Compumedicus, Melbourne, Vic, Australia or Alice4TM; Healthdyne, International, USA), to determine the stages of sleep from 4 electroencephalogram (EEG) channels of (C3/A2-O2/A1, C4/A1-O1/A2), 2 electrooculogram (EOG) channels (ROC/LOC), and the submental electromyogram (EMG). Respiratory variables were monitored using 


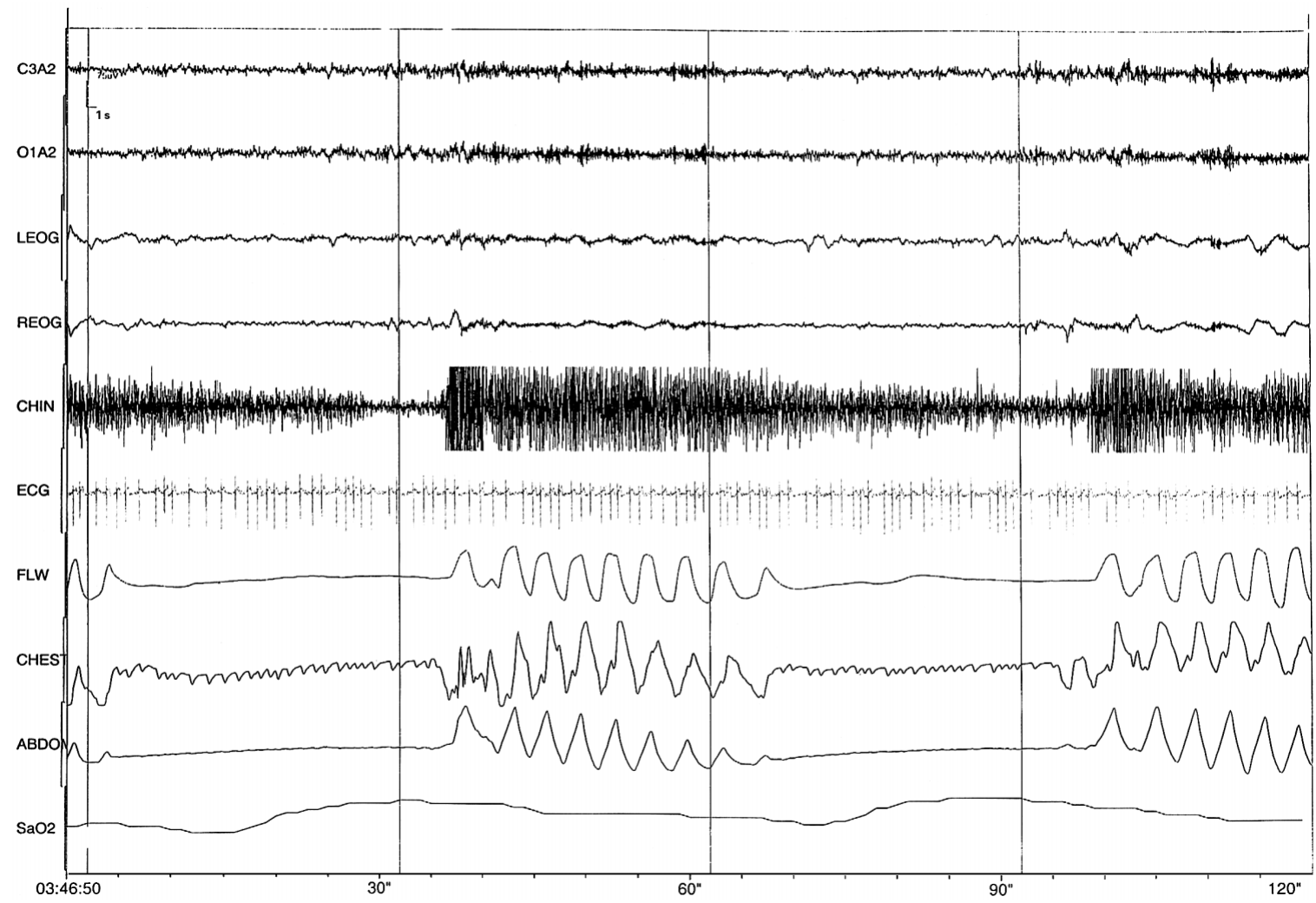

Fig 1. Polysomonographic recording shows central sleep apnea-hypopnea syndrome (CSAHS) in a patient with dilated cardiomyopathy (DCM). C3A2, O1A2: electroencephalogram(EEG); ECG, electrocardiogram; EOG, electrooculogram; CHIN, chin electromyogram, ECG; FLW, nasal airflow; CHEST, thoracic movement; ABDO, abdominal movement; $\mathrm{SaO}_{2}$, arterial oxygen saturation.

abdominal and thoracic impedance bands to assess the chest wall movement and a diaphragmatic EMG electrode. Arterial oxygen saturation $\left(\mathrm{SaO}_{2}\right)$ was continuously measured with a finger pulseoximeter. Nasal and oral airflow was recorded with a thermistor or pressure sensor. The bilateral tibialis anterior EMG and the electrocardiogram (ECG) were recorded during PSG.

Sleep was scored over 30-s intervals using the criteria of Rechtshaffen and Kales! ${ }^{14}$ Apnea was defined as cessation of inspiratory airflow for $10 \mathrm{~s}$ or more. Obstructive apnea was defined as the absence of airflow in the presence of rib cage and abdominal excursions. Central apnea was defined as the absence of rib cage and abdominal excursions with an absence of airflow. Hypopnea was defined as a reduction of airflow lasting $10 \mathrm{~s}$ or more and associated with decrease of $3 \%$ or more in the $\mathrm{SaO}_{2}$ of hemoglobin or with arousal. The hourly number of episodes of apnea and hypopnea was calculated as the apnea-hypopnea index (AHI). CheyneStokes respiration (CSR) was defined as a gradual waxing and waning of respiration followed by central apnea or central hypopnea.

\section{Other Studies}

As an objective measure of left ventricular (LV) contractile function, the LV ejection fraction (LVEF) was measured by left ventriculography or transthoracic echocardiography when the patients were admitted to hospital for the assessment of cardiomyopathy and sleep-disordered breathing.

\section{Classification of Patients}

Patients were divided into those with obstructive sleep apnea-hypopnea syndrome (OSAHS), who had an AHI of 5 or more, those with central sleep apnea-hypopnea syndrome (CSAHS; all of whom had an obstructive AHI $<5 / \mathrm{h}$ ), and those without sleep apnea-hypopnea syndrome (SAHS). If more than $50 \%$ of the episodes of sleep-disordered breathing were central the patient was defined as having CSHAS, but was defined as OSAHS if $>50 \%$ of events were obstructive. All patients were classified as OSAHS, CSAHS, or non-SAHS.

\section{Statistical Analysis}

We used the Mann-Whitney U test to assess significance differences between HCM patients with OSAHS and those without SAHS. Because there were 3 groups of DCM patients, Fisher's protected least significant difference test was used to compare the significance of differences among these groups. A p-value $<0.05$ was considered statistically significant. All descriptive data are expressed as mean \pm SD. Statistical analysis was done with StatView software Version 5.0 (SAS institute Inc, Cary, NC, USA).

\section{Results}

The 35 patients with cardiomyopathy included 20 with DCM and 15 with HCM. The DCM patients were divided into those with CSAHS $(n=10,50 \%)$, OSAHS $(n=6,30 \%)$, and those with non-SAHS $(n=4,20 \%)$. A representative polysomnographic recording of a DCM patient with CSAHS is presented in Fig 1. Five of the 10 DCM patients 


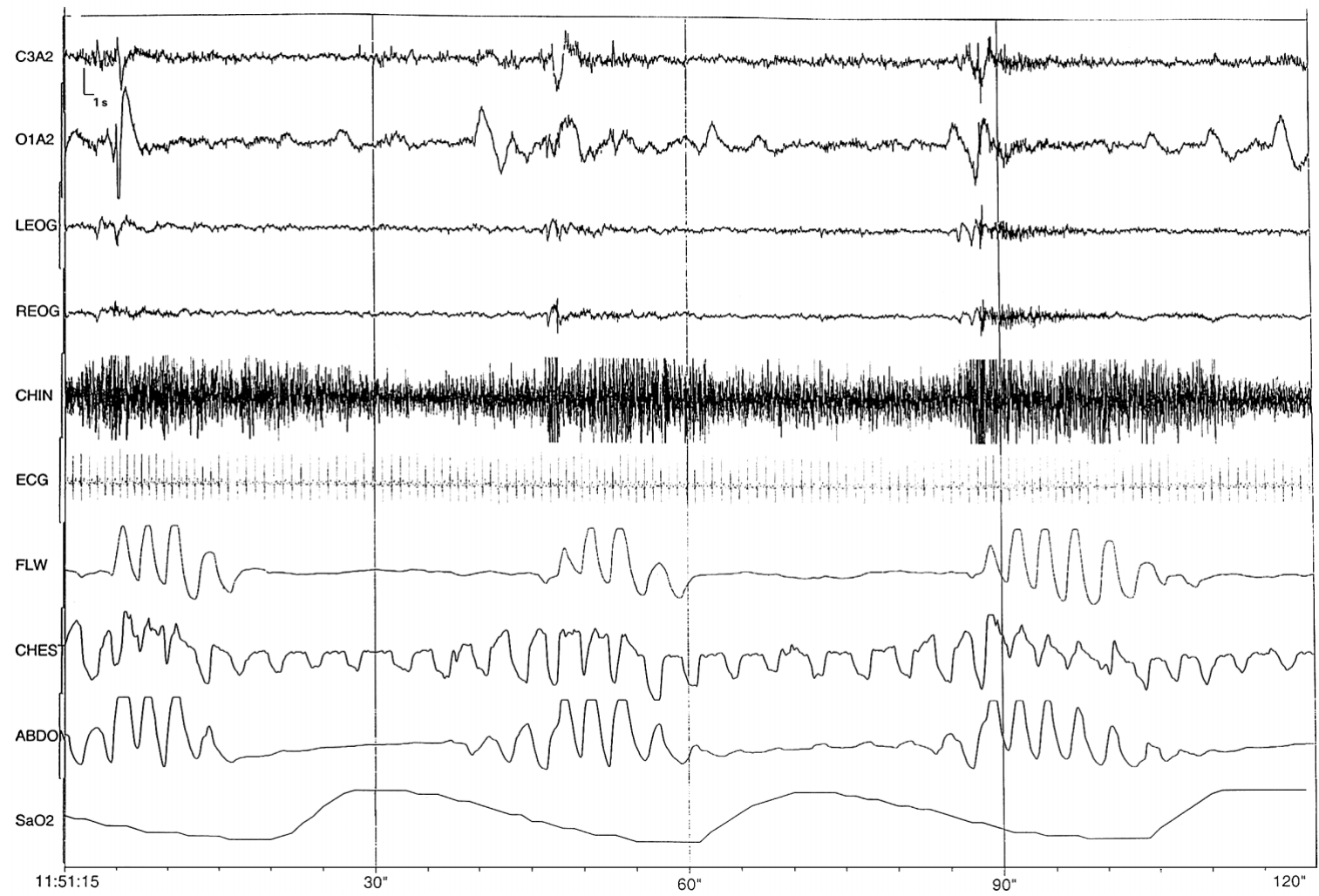

Fig 2. Polysomnographic recording shows obstructive sleep apnea-hypopnea syndrome (OSAHS) in a patient with hypertrophic cardiomyopathy (HCM). C3A2, O1A2: electroencephalogram (EEG); ECG, electrocardiogram; EOG, electrooculogram; CHIN, chin electromyogram, ECG; FLW, nasal airflow; CHEST, thoracic movement; ABDO, abdominal movement; $\mathrm{SaO}_{2}$, arterial oxygen saturation.

Table 1 Clinical Characteristics of the Patients With Cardiomyopathy

\begin{tabular}{|c|c|c|c|c|c|}
\hline & \multicolumn{3}{|c|}{$D C M$} & \multicolumn{2}{|c|}{$H C M$} \\
\hline & CSAHS & OSAHS & non-SAHS & OSAHS & non-SAHS \\
\hline Patients, $n(\%)$ & $10(50)$ & $6(30)$ & $4(20)$ & $7(47)$ & $8(53)$ \\
\hline Age (years) & $48.4 \pm 14.2$ & $43.3 \pm 9.5$ & $47.0 \pm 14.0$ & $48.4 \pm 8.3$ & $47.8 \pm 15.7$ \\
\hline Weight ( $k g$ ) & $64.1 \pm 14.9$ & $81.3 \pm 19.3$ & $59.7 \pm 12.8$ & $75.1 \pm 11.4^{\# \#}$ & $54.6 \pm 11.2$ \\
\hline Height $(\mathrm{cm})$ & $162.1 \pm 7.2$ & $166.0 \pm 5.2$ & $166.5 \pm 6.2$ & $164.9 \pm 2.1$ & $157.3 \pm 9.1$ \\
\hline$B M I\left(\mathrm{~kg} / \mathrm{m}^{2}\right)$ & $24.2 \pm 4.0$ & $29.3 \pm 5.8^{*}$ & $21.3 \pm 3.1$ & $27.6 \pm 3.8^{\#}$ & $22.0 \pm 4.0$ \\
\hline$A H I(/ h)$ & $18.0 \pm 8.1$ & $26.9 \pm 26.2 * *$ & $2.5 \pm 1.6$ & $28.1 \pm 29.1^{\# \#}$ & $1.5 \pm 1.2$ \\
\hline Mean $\mathrm{SpO}_{2}(\%)$ & $96 \pm 2$ & $95 \pm 4$ & $97 \pm 2$ & $94 \pm 2$ & $96 \pm 2$ \\
\hline Lowest $\mathrm{SpO}_{2}(\%)$ & $79 \pm 11$ & $76 \pm 16$ & $92 \pm 3$ & $74 \pm 17$ & $84 \pm 9$ \\
\hline$S B P(m m H g)$ & $125 \pm 12$ & $123 \pm 13$ & $124 \pm 13$ & $132 \pm 12$ & $128 \pm 11$ \\
\hline$D B P(m m H g)$ & $73 \pm 9$ & $71 \pm 13$ & $76 \pm 4$ & $77 \pm 6$ & $73 \pm 6$ \\
\hline $\operatorname{LVEF}(\%)$ & $32 \pm 18$ & $34 \pm 9$ & $27 \pm 7$ & $77 \pm 10$ & $78 \pm 3$ \\
\hline \multicolumn{6}{|l|}{ NYHA classification } \\
\hline Classes I and II, $n(\%)$ & $5(50)$ & $5(83)$ & $3(75)$ & $7(100)$ & $7(88)$ \\
\hline Classes III and IV, $n(\%)$ & $5(50)$ & $1(17)$ & $1(25)$ & $0(0)$ & $1(13)$ \\
\hline
\end{tabular}

CSAHS, central sleep apnea-hypopnea syndrome; OSAHS, obstructive sleep apnea-hypopnea syndrome; non-SAHS, non sleepapnea hypopnea syndrome; BMI, body mass index; AHI, apnea hypopnea index; SBP, systolic blood pressure; DBP, diastolic pressure; LVEF, left ventricular ejection fraction; NYHA, New York Heart Association. Values are mean $\pm S D$.

${ }^{*} p<0.05$ vs CSAHS and non-SAHS, ${ }^{* *} p<0.05$ vs non-SAHS, ${ }^{*} p<0.05$ vs non-SAHS, ${ }^{*} p<0.01$ vs non-SAHS.

with CSAHS had CSR. DCM patients with CSAHS had a lower $\mathrm{PaCO}_{2}(36.7 \pm 2.7 \mathrm{mmHg})$ than those without CSAHS ( $p<0.01$ vs DCM patients with OSAHS: $41.8 \pm 1.8 \mathrm{mmHg}$, $\mathrm{p}<0.01$ vs DCM patients without SAHS: $42.3 \pm 1.8 \mathrm{mmHg}$ ). HCM patients were divided into those with OSAHS $(\mathrm{n}=7$, $47 \%)$ and those without SAHS $(\mathrm{n}=8,53 \%)$. Fig 2 shows OSAHS in a patient with HCM. The LVEF of the DCM patients was lower than that of the HCM patients $(32 \pm 14$ vs $78 \pm 7 \%, \mathrm{p}<0.001)$. The clinical data are summarized in
Table 1.

DCM patients with OSAHS were significantly more obese and had a greater body mass index (BMI) (29.3 \pm $5.8 \mathrm{~kg} / \mathrm{m}^{2}$ ) than DCM patients without OSAHS ( $<<0.05 \mathrm{vs}$ DCM patients with CSAHS: $24.2 \pm 4.0 \mathrm{~kg} / \mathrm{m}^{2}, \mathrm{p}<0.05$ vs DCM patients without SAHS: $21.3 \pm 3.1 \mathrm{~kg} / \mathrm{m}^{2}$ ). There were no significant differences of age, systolic blood pressure (SBP), diastolic blood pressure (DBP) and LVEF between the 3 groups of DCM patients. One of the obese DCM 
patients with severe OSAHS was treated with continuous positive airway pressure (CPAP). His AHI decreased from 56.6 to $6.7 / \mathrm{h}$.

The HCM patients were divided into 2 subgroups. The patients with OSAHS weighed more than those without SAHS $(75.1 \pm 11.4$ vs $54.6 \pm 11.2 \mathrm{~kg}, \mathrm{p}<0.01)$. Similarly, the BMI of the OSAHS patients was greater than that of those without SAHS $\left(27.6 \pm 3.8\right.$ vs $\left.22.0 \pm 4.0 \mathrm{~kg} / \mathrm{m}^{2}, \mathrm{p}<0.05\right)$. However, the age, SBP, DBP, and LVEF did not differ significantly between these 2 groups. We treated an obese HCM patient with severe OSAHS with CPAP, which resulted in a reduction in the AHI (from 49.3 to $6.4 / \mathrm{h}$ ).

\section{Discussion}

In the present study sleep-disordered breathing was frequently seen in DCM or HCM patients. DCM patients with stable heart failure had 2 types of sleep-disordered breathing: OSAHS and CSAHS. We found that OSAHS occurred significantly more often in the obese DCM patients, who had a larger BMI than either those with CSAHS or those without SAHS. Young et al reported that obesity is an important risk factor for OSAHS 15 and Javaheri et al showed that BMI was significantly and positively correlated with OSAHS in patients with stable chronic heart failure 16,17 Even in patients with DCM it seems that obesity could be a factor in the occurrence of OSAHS, which is caused by anatomical airway obstruction.

The diagnosis of CSHAS was made in half of the DCM patients, but not in any of the HCM patients. Half of the DCM patients with CSAHS were classified in New York Heart Association (NYHA) classes III and IV. Javaheri et al reported that a low LVEF was associated with increased prevalence of CSHAS ${ }^{18}$ and Thalhofer et al reported that 6 of 8 patients with DCM (NYHA III or IV) had CSAHS and CSR 10 Another study by Javaheri et al has shown that CSAHS was common in patients diagnosed as idiopathic cardiomyopathy with chronic heart failure ${ }^{16}$ Wilcox et al showed that DCM patients with severe LV dysfunction had CSAHS 11 and other researchers have shown that a low LVEF is associated with CSAHS or CSR in patients with DCM6-9

Our results showed there was no significant difference in the LVEF of DCM patients with CSAHS and those without SAHS, but DCM patients with CSAHS had a lower $\mathrm{PaCO}_{2}$ than those without CSAHS. The mechanism by which DCM patients with poor cardiac function develop CSAHS is unclear, but it seems that CSAHS is associated with not only a low LVEF but also another factor in DCM patients. Enhanced sensitivity to carbon dioxide may predispose patients with heart failure to suffer from CSAHS 19 Thalhofer et al reported that CSR is seen in patients with DCM and they demonstrated that some patients still had CSR after normalization of cardiac function by heart transplantation. ${ }^{20}$ It seems that a low $\mathrm{PaCO}_{2}$ is a significant risk factor for CSAHS in patients with chronic heart failure? 1 Tkacova et al reported that CSHAS is associated with a reduction in $\mathrm{PaCO}_{2}$, causing a concurrent lengthening of circulation,2 and Naughton et al also reported that a low $\mathrm{PaCO}_{2}$ was a key predisposing factor for CSAHS in chronic heart failure ${ }^{23}$ Hypocapnia leads to high left ventricular filling pressures and pulmonary congestion, which causes hyperventilation through stimulation of pulmonary vagal irritant receptors ${ }^{24}$ It is also possible that DCM patients with CSAHS may have altered function of the central res- piratory center under the long-term influence of hypocapnia or hypoxemia.

In the present study we demonstrated that HCM patients with a larger BMI had OSAHS but not CSAHS. Their NYHA classes were I or II. Although DCM patients tend to have a low LVEF, the LVEF of HCM patients is normal to supranormal. It is possible that, depending on their BMI, the patients with HCM have the potential to develop OSAHS, which is caused by upper airway obstruction related to obesity. However, because we selected HCM patients who are not at the end stage, we could not assess the prevalence of CSAHS. Further studies are needed with respect to the relationship between HCM patients with low LVEF and CSAHS.

We confirmed the effect of CPAP therapy on a DCM patient with respect to AHI. Malone et al reported that OSAHS makes LV function worse in DCM patients whereas nasal CPAP can lead to improvements of symptoms and cardiac function? The reduction in afterload by using CPAP has been reported in DCM patients with $\mathrm{OSAHS}^{3}$ and although the indication of CPAP for patients with cardiomyopathy has not been established, it seems to be an effective therapy for obese DCM patients with OSAHS.

We tried CPAP on an obese HCM patient and found there was a marked reduction in the AHI. Patients with OSAHS have a high risk of sudden death,25 which is also a common complication of $\mathrm{HCM}^{26,27}$ The magnitude of the LV hypertrophy has been considered a risk factor for sudden death in HCM patients ${ }^{28}$ Complications of OSAHS may induce further LV hypertrophy and thereby increase the risk of sudden death. Further studies are required to elucidate the potential benefits of CPAP for HCM patients with sleep-disordered breathing.

\section{References}

1. Richardson P, McKenn W, Bristow M, Maisch B, Mautner B, O'Connell J, et al. Report of the 1995 World Health Organization/International Society and Federation of Cardiology Task Force on the Definition and Classification of cardiomyopathies. Circulation 1996; 93: $841-842$.

2. Malone S, Liu PP, Holloway R, Rutherford R, Xie A, Bradley TD. Obstructive sleep apnoea in patients with dilated cardiomyopathy: Effects of continuous positive airway pressure. Lancet 1991; 338: 1480-1484.

3. Tkacova R, Rankin F, Fitzgerald FS, Floras JS, Bradley TD. Effects of continuous positive airway pressure on obstructive sleep apnea and left ventricular afterload in patients with heart failure. Circulation 1998; 98: 2269-2275

4. Takasaki Y, Orr D, Popkin J, Rutherford R, Liu P, Bradley TD. Effect of nasal continuous positive airway pressure on sleep apnea in congestive heart failure. Am Rev Respir Dis 1989; 140: 1578-1584.

5. Thomas RJ. The cardiomyopathy of obstructive sleep apnea. Ann Intern Med 1996; 125: 425.

6. Lanfranchi PA, Braghiroli A, Bosimini E, Mazzuero G, Colombo R, Donner CF, et al. Prognostic value of nocturnal Cheyne-Stokes respiration in chronic heart failure. Circulation 1999; 99: 1435-1440.

7. Solin P, Roebuck T, Swieca J, Walters EH, Naughton MT. Effects of cardiac dysfunction on non-hypercapnic central sleep apnea. Chest 1998; 113: $104-110$.

8. Tkacova R, Hall MJ, Liu PP, Fitzgerald FS, Bradley TD. Left ventricular volume in patients with heart failure and Cheyne-Stokes respiration during sleep. Am J Respir Crit Care Med 1997; 156: $1549-1555$

9. Andreas S, Hagenah G, Moller C, Werner GS, Kreuzer H. CheyneStokes respiration and prognosis in congestive heart failure. Am J Cardiol 1996; 78: 1260-1264.

10. Thalhofer S, Dorow P, Schuler S, Hetzer R. Oxygen saturation and sleep structure in patients with dilated cardiomyopathy. Pneumologie 1991; 45(Suppl 1): 259-260.

11. Wilcox I, McNamara SG, Willson GN, Peper A, Pope P, Grunstein $\mathrm{R}$, et al. Is sleep apnea a new prognostic marker in heart failure? 
Circulation 1995; 92: I-274 (abstract).

12. Shiomi T, Kobayashi T, Mizutani K, Yano Y, Maekawa K, Kawai H, et al. A study on a new detector for sleep apnea in various cardiovascular diseases with special reference to its usefulness of a respiratory Holter's monitoring system. J Aichi Med Univ Assoc 1987; 55: 357366.

13. Maron BJ. Hypertrophic cardiomyopathy: A systematic review. JAMA 2002; 287: 1308 - 1320 .

14. Rechtshaffen A, Kales A. A manual of standardised terminology, techniques and scoring system for sleep stages of human subjects. Washington DC: US Government Printing Office, 1968.

15. Young T, Palta M, Dempsey J, Skatrud J, Weber S, Badr S. The occurrence of sleep-disordered breathing among middle-aged adults. N Engl J Med 1993; 328: 1230-1235.

16. Javaheri S, Parker TJ, Liming JD, Corbett WS, Nishiyama H, Wexler $\mathrm{L}$, et al. Sleep apnea in 81 ambulatory male patients with stable heart failure: Types and their prevalences, consequences, and presentations. Circulation 1998; 97: 2154-2159.

17. Javaheri S, Parker TJ, Wexler L, Michaels SE, Stanberry E, Nishyama H, et al. Occult sleep-disordered breathing in stable congestive heart failure. Ann Intern Med 1995; 122: 487-492.

18. Javaheri S. Central sleep apnea-hypopnea syndrome in heart failure: Prevalence, impact, and treatment. Sleep 1996; 19(Suppl 10): S229_ S231.

19. Javaheri S. A mechanism of central sleep apnea in patients with heart failure. $N$ Engl J Med 1999; 341: 949-954.

20. Thalhofer SA, Kiwus U, Dorow P. Influence of orthotopic heart transplantation on breathing pattern disorders in patients with dilated cardiomyopathy. Sleep Breath 2000; 4: 121-126.

21. Sin DD, Fitzgerald F, Parker JD, Newton G, Floras JS, Bradley TD. Risk factors for central and obstructive sleep apnea in 450 men and women with congestive heart failure. Am J Respir Crit Care Med 1999; 160: $1101-1106$.

22. Tkacova R, Niroumand M, Lorenzi-Filho G, Bradley TD. Overnight shift from obstructive to central apneas in patients with heart failure: Role of $\mathrm{PCO}_{2}$ and circulatory delay. Circulation 2001; 103: $238-$ 243.

23. Naughton M, Benard D, Tam A, Rutherford R, Bradley TD. Role of hyperventilation in the pathogenesis of central sleep apneas in patients with congestive heart failure. Am Rev Respir Dis 1993; 148: 330-338.

24. Solin P, Bergin P, Richardson M, Kaye DM, Walters EH, Naughton MT. Influence of pulmonary capillary wedge pressure on central apnea in heart failure. Circulation 1999; 99: 1574-1579.

25. Rossner S, Lagerstrand L, Persson HE, Sachs C. The sleep apnoea syndrome in obesity: Risk of sudden death. J Intern Med 1991; 230: $135-141$.

26. Maron BJ, Olivotto I, Spirito P, Casey SA, Bellone P, Gohman TE, et al. Epidemiology of hypertrophic cardiomyopathy-related death: Revisited in a large non-referral-based patient population. Circulation 2000; 102: $858-864$.

27. Maron BJ. Hypertrophic cardiomyopathy. Lancet 1997; 350: $127-$ 133.

28. Spirito P, Bellone P, Harris KM, Bernabo P, Bruzzi P, Maron BJ. Magnitude of left ventricular hypertrophy and risk of sudden death in hypertrophic cardiomyopathy. N Engl J Med 2000; 342: 1778-1785. 